\title{
Photoelectron-Auger electron coincidence spectroscopy of free molecules: new experiments
}

Volker Ulrich $^{1}$, Silko Barth ${ }^{1}$, Toralf Lischke ${ }^{1}$, Sanjeev Joshi ${ }^{1}$, Tiberiu Arion ${ }^{1}$, Melanie Mucke ${ }^{1}$, Marko Förstel $^{1,2}$, Alex M. Bradshaw ${ }^{1,3}$, and Uwe Hergenhahn ${ }^{1, *}$

1. Max-Planck-Institut für Plasmaphysik (IPP), EURATOM Association, Boltzmannstr. 2, 85748 Garching, Germany

2. Max-Planck-Institut für Kernphysik, Saupfercheckweg 1, 69117 Heidelberg, Germany

3. Fritz-Haber-Institut der Max-Planck-Gesellschaft (FHI), Faradayweg 4-6, 14195 Berlin, Germany

Dedicated to the memory of Professor Kai Siegbahn

\section{Abstract}

Photoelectron-Auger electron coincidence spectroscopy probes the dicationic states produced by Auger decay following the photoionization of core or inner valence levels in atoms, molecules or clusters. Moreover, the technique provides valuable insight into the dynamics of core hole decay. This paper serves the dual purpose of demonstrating the additional information obtained by this technique compared to Auger spectroscopy alone as well as of describing the new IPP/FHI apparatus at the BESSY II synchrotron radiation source. The distinguishing feature of the latter is the capability to record both the photoelectron and Auger electron with good energy and angle resolution, for which purpose a large hemispherical electrostatic analyser is combined with several linear time-of-flight spectrometers. New results are reported for the K-shell photoionization of oxygen $\left(\mathrm{O}_{2}\right)$ and the subsequent K-VV Auger decay. Calculations in the literature for non-coincident $\mathrm{O}_{2}$ Auger spectra are found to be in moderately good agreement with the new data.

\section{PACS}

07.81.+a, 07.85.Qe, 29.30.Dn, 32.80.Hd, 33.80.Eh

\footnotetext{
${ }^{*}$ Corresponding author, Mail address: c/o Helmholtz-Zentrum Berlin, Albert-Einstein-Str. 15, 12489 Berlin, Germany. Electronic address: uwe.hergenhahn@ipp.mpg.de.
} 


\section{Introduction}

Auger decay is widely used as an element-specific probe of material composition, in particular, in surface analysis [1]. In molecules, the Auger spectrum also contains information about the electronic and nuclear structure of the final (dicationic) states of the radiationless decay process. Some of the very first high resolution Auger spectra of molecules can be found in Kai Siegbahn's second book [2]. The Auger spectrum of an atom of a particular element, characterized by its nuclear charge $Z$, has a specific appearance according to the molecular environment in which the atom finds itself. However, a detailed understanding of the spectra is difficult because of the large number of dicationic states that are relevant in a molecule [3] and because of the influence of the nuclear dynamics in the decay process [4][5]. Moreover, the question arises as to whether Auger decay in molecules is a local process, i.e., whether the initial inner shell vacancy is filled only by valence electrons originating from the same atom [6][7][8]. A further complication arises for all but the simplest molecules, since the Auger emission from different atoms of the same sort (i.e. with identical $Z$ ) - should they be present will overlap. Conventional (non-coincident) electron spectroscopy is typically not able to disentangle the contributions of such atoms, or of different vibrational levels associated with the same final state.

Electron-electron coincidence spectroscopy has the potential to greatly enhance the amount of information which can be extracted from photoelectron and Auger electron spectroscopy. In the following we consider such processes after excitation with small bandwidth soft X-radiation as provided by undulators at an electron storage ring. Early photoelectron-Auger electron coincidence experiments were concerned with bulk and adsorbate systems [9][10]; more recent work on bulk systems has been reviewed by Stefani et al. [11]. The technique was first applied to gas phase systems by Kämmerling and Schmidt [12]. Useful comments on these early results can be found in Ref. [13]. Application of the electron-electron coincidence technique to free molecules demonstrated the possibility of identifying the contributions of satellite autoionization to the main KVV Auger spectrum in $\mathrm{N}_{2}$ [14]. Moreover, a study by Viefhaus et al. [15] showed how the resolution barrier in core level photoelectron spectroscopy dictated by the natural lifetime broadening can be overcome. This can be understood as follows: the energy balance of the process reads $E_{\mathrm{fi}}=h \nu+E_{0}-\varepsilon_{\mathrm{ph}}-\varepsilon_{\mathrm{au}}$, where $E_{\mathrm{fi}}$ and 
$E_{0}$ are the final (dicationic) and the initial (neutral ground) state energies, $\varepsilon_{\mathrm{ph}}$ and $\varepsilon_{\mathrm{au}}$ kinetic energies of the photo- and the Auger electron, and $h v$ the photon energy. Thus, if the final state is long lived and the bandwidth, with which $h v, \varepsilon_{\mathrm{ph}}$ and $\varepsilon_{\mathrm{au}}$ are selected, is sufficiently low, structures can be discerned in the coincidence spectrum below the limit of the lifetime broadening (Fig. 1). For a number of systems, Coster-Kronig decays from inner valence states have been investigated thus [16][17][18]. In future detailed studies of the nuclear dynamics in the intermediate (core ionized) state may therefore become possible [19][20]. This topic has been of great interest in the analogous situation of resonant Auger decay, where, due to the fact that only one electron is escaping from the molecule, sub-natural resolution has been achieved [21][22] without the need of a coincidence set-up.

In this article we discuss not only the technique of photoelectron-Auger electron coincidence spectroscopy but also describe a new apparatus for the detection of electron-electron pairs with superior energy resolution. The design of the latter is based on the combination of a large electrostatic hemispherical electron analyser with several medium-sized linear time-of-flight (TOF) spectrometers. The construction of the apparatus and the details of the data acquisition procedure are first explained, some principal features of the spectra are then described, and finally some new experimental data for molecular oxygen are shown. First results obtained with this apparatus (for CO) have been published elsewhere [23], but are shown again for illustrative purposes. Together with the description of our apparatus we also include a critical comparison with other spectrometers for coincident electron detection. A complete review of photoelectron-Auger electron coincidence spectroscopy is, however, outside the scope of this publication.

\section{Experimental Set-up}

A sketch of the main apparatus parts is shown in Fig. 2. A spherical vacuum chamber [24] holds a hemispherical analyser of $200 \mathrm{~mm}$ nominal radius (Scienta ES 200, [25]) and several TOF spectrometers. The Scienta analyser has been fitted with a fast position-resolving anode based on a delay line (Roentdek, Frankfurt, Germany), in order to make it capable of performing coincidence data acquisition. The properties of this system have been discussed [26]. The Scienta analyser is positioned at the so-called magic angle of $54.7^{\circ}$ with respect to the horizontal. Therefore, with horizontally 
linearly polarized radiation, the non-coincident differential cross sections recorded are proportional to the total cross section within the electric dipole approximation. No such analogous pair of angles is known for two-electron emission. The TOF analysers therefore have been placed at positions which are convenient from the point of view of the chamber geometry.

All data covered here were recorded at the BESSY II synchrotron radiation source (Berlin, Germany). The oxygen spectra shown in section IV were measured at the UE52-SGM beamline [27]. This beamline is equipped with an undulator of the Apple-II type [28], which was set to produce vertically linearly polarized radiation. The $1200 \mathrm{l} / \mathrm{mm}$ grating was used with a resolution selected as $140 \mathrm{meV}$ (estimated from ray-tracing calculations). The photon energy was set at $551.85 \mathrm{eV}$, with about $\pm 0.2 \mathrm{eV}$ calibration uncertainty. Sample gas was leaked into the chamber up to a background pressure of $3 \times 10^{-6} \mathrm{mbar}$, limited in the first instance by the ratio of true to random coincidences (see below).

\section{A. Time-of-Flight Analysers}

Time-of-flight analysers (TOFs) for electron spectroscopy have been described e.g. by Hemmers et al., Feulner et al. and Becker et al. [29][30][31][32]. The decision to use this principle in our set-up was made because TOFs allow a range of energies to be covered in parallel, with modest construction effort and at a good energy resolution. Our design consists of a conical drift tube of $110 \mathrm{~mm}$ length which is followed by a second, cylindrical drift tube. The entrance is formed by a spherical diaphragm (4 mm diameter) at a distance of $30 \mathrm{~mm}$ to the interaction zone. Both drift regions can be set to different electric potentials in order to improve the resolution by retarding or, alternatively, the transmission for low kinetic energy electrons by accelerating. Often, voltages can be selected such that electron lens effects formed in the transit regions between the different potentials increase the angular acceptance, and by that the detected intensity for an energy region of interest. The cylindrical drift tube is terminated by a copper mesh, and electrons are then accelerated onto a triple microchannel plate (MCP) stack (MCP outer diameter $34.5 \mathrm{~mm}$ ). The holder for the MCPs is a simplified version of the design by Snell et al. [33]. The MCPs can be dismounted from the vacuum chamber independently of the TOFs themselves. Most conducting parts are made of aluminum, insulating parts of polyetheretherketone (PEEK). 
The total length of the drift section (centre of interaction to mesh) can be varied, and amounts to either 228.5 or $268.5 \mathrm{~mm}$. From geometrical considerations of a point-like interaction region a solid angle of acceptance of about 14 msR follows, which is determined both by the MCP active area and the entrance aperture. In all measurements shown here the shorter drift section was used.

For these analysers, we have demonstrated an energy resolution of $70 \mathrm{meV}$ for photoelectrons of $8 \mathrm{eV}$ kinetic energy, retarded with -4.5/-7.6 V in the conical and cylindrical section, resp. If detection of electrons with low kinetic energy is of interest, a positive (acceleration) voltage can be used in the drift tube, and we have demonstrated a flat transmission function down to at least $0.3 \mathrm{eV}$ with acceleration values of $+1 /+2 \mathrm{~V}$. The energy resolution of the analysers is strongly influenced by the effective size and shape of the interaction region. From geometrical considerations, electrons produced by the synchrotron radiation (SR) beam along a path length of the order of $10 \mathrm{~mm}$ are accepted in the TOFs, which leads to a degradation of the resolution as electron trajectories from non-central source points differ in length. This effect is the worse the smaller the angle formed by the TOF and the SR beam. For an analyser at $45^{\circ}$, a difference of about $4 \%$ in length is estimated. On the other hand, the sample is formed by an effusive gas jet leaking in via a $d=0.8 \mathrm{~mm}$ copper capillary, which leads to a strong peaking of the gas density at the point where the TOF middle axes intersect [34].

A conservative estimate of the true TOF resolution can be found from ray-tracing simulations of the electron trajectories, which are presented in Fig. 3. Simulations where carried out with the SimIon 8.0 programme (SIS, Ringoes, NJ. USA). For the simulations, electrons with kinetic energies of $7.7 \mathrm{eV}$, $8.0 \mathrm{eV}$ and $8.3 \mathrm{eV}$ were considered. Trajectories for 90000 electrons were calculated for each source point and each kinetic energy, distributed homogeneously in a filled cone with a half angle of $20^{\circ}$. For consistency, the retarding voltages of the two stages of the spectrometer were set to $-4.5 /-7.6 \mathrm{~V}$, similar to the experiment. The simulated spectra present the ideal case of a point-like interaction region (dotted curve), and the more realistic case of a pencil shaped electron source of $10 \mathrm{~mm}$ length, $2 \mathrm{~mm}$ diameter and an inclination of the spectrometer with respect to the TOF middle axis of $44.4^{\circ}$ (solid curve). As expected, the resolution of the spectrometer worsens on moving from the ideal case 
to realistic conditions, but even then remains better than $70 \mathrm{meV}$ approx. Electronic contributions to the resolution, such as jitter of the timing signals, were not taken into account in the simulation.

The simultaneous operation of several TOF analysers is advantageous, since the true coincidence rate scales with the solid angle (TOF-Scienta coincidences) or the square of the solid angle (TOF-TOF coincidences) covered. From geometrical constraints given by the vacuum recipient, including the Scienta analyser and an expansion chamber to produce free molecular jets and cluster jets [24], the TOF analysers had to be mounted in a vertical plane, pointing downward (see Fig. 2). Some remarks concerning angular effects by this choice are in order. Photoelectrons excited by linearly polarized light normally have a propensity for emission along the polarization axis (positive $\beta$ parameter) [32]. Synchrotron radiation from conventional beamlines is linearly polarized in the storage ring plane, which leads to unfavorable detection efficiencies in our geometry. However, the flexibility of the Apple-II undulator design allows to also produce vertically linearly polarized radiation [28]. In all studies reported here, this setting was used to increase the number of registered photoelectrons. The hemispherical analyser by that is not placed at the magic angle anymore. Since it typically is used to detect the Auger electrons, which in a non-coincident experiment have a rather isotropic angular distribution, this is less of a concern. For the triply differential cross section in sequential photodouble-ionization no general picture has emerged so far. Photoelectron-Auger electron coincidence experiments on atoms suggest that back-to-back emission of the two electrons is favourable when the photoelectron is emitted along the (linear) polarization axis, but chiral, more complicated angular correlation patterns have been observed when the photoelectron analyser is moved away from the polarization direction [13][35][36]. Contrary to those experiments, our apparatus does not allow a relative rotation of the analysers. Detection of the photoelectrons under a variable angle with respect to the polarization direction could be achieved by rotating the polarization ellipse of the synchrotron radiation however, which is possible at some undulators [37]. As our analysers, except for the central one, are placed outside of the dipole plane, they are sensitive to angular distribution terms from higher multipole orders in the photon-molecule interaction. Most recent studies for two systems found an only small influence from this mechanism [38][39]. 
In the measurements shown here the signals of all TOF analysers were added to increase the coincidence rate. The $66.6^{\circ}$ analyser was not used.

\section{B. Data acquisition}

Electron-electron coincidence signals in the two types of analyser could be recorded in a straightforward manner when the separation of two successive synchrotron radiation pulses was larger than the typical time-of-flight for low kinetic energy electrons in the TOF analyser. The latter is of the order of 300 ns. The use of the single bunch mode of BESSY would then be required to meet this condition. Due to the small solid angle accepted by the detectors, however, either the number of accidental coincidences would then be unacceptably large or the time required for data acquisition would be unpractical. We have therefore chosen to record coincidences during multi-bunch (normal) operation. Under these conditions, synchrotron radiation is delivered in pulses of 20-30 ps length with a spacing of 2 ns [40]. A train of 350 electron bunches is followed by a dark gap of 100 ns length, in the middle of which a single electron bunch is injected, resulting in the so-called hybrid peak [41]. Thus, the non-coincident electron time-of-flight spectra excited by the different bunches overlap and cannot be disentangled in a simple time-of-flight measurement. Only the spectrum excited by the hybrid peak can often be separated, and is seen either in the dark gap or on top of a diffuse background of slow electrons. This serves as a valuable calibration. Typical non-coincident time-of-flight data (for C 1s emission in CO) are shown in Fig. 4, and will be discussed in the next section.

A schematic diagram of the data acquisition electronics is shown in Fig. 5. In the first version of the electronics, shown in the figure, only four TDC channels were available. Therefore, signals from all TOF analysers were multiplexed by OR gates. Signal path lengths for the different analysers were adjusted to within one ns. In the same way, the four signals from the delay line anode were collected in two TDC channels. Signal pairs were separated by use of a cable delay in two of the four signal paths. 
For the coincident detection of an electron pair by one of the TOFs and the hemispherical analyser, the problem of implicit convolution with the BESSY fill pattern can be overcome by referring the TOF arrival time not to the bunch clock of BESSY, but to the time of electron detection in the hemispherical analyser. Since the latter filters electrons with a selected energy, it can be expected that these have an approximately fixed time-of-flight in this instrument. In practice, the hemispherical analyser is set to detect (fast) Auger electrons, such that comparatively large pass energies (typically 75-300 eV) can be used. This helps in the reduction of the time-of-flight spread for the trajectories within the analyser, as discussed in detail below (see also [26][42][43]). Thus, referring the photoelectron arrival times to the Auger arrival times allows the experiment to be carried out in multibunch mode, with a greatly improved true-to-random ratio, but at the expense of a slight reduction of time resolution, and thus energy resolution of the photoelectron.

\section{Typical time-of-flight data}

It is instructive to examine some typical data sets. To demonstrate the resolution achieved in the TOF detectors, we refer first to the $\mathrm{C} 1$ s spectrum of $\mathrm{CO}$, as recorded in the first experiments with the new apparatus [23]. Carbon K-shell photoionization of CO, at a threshold energy of $296.07 \mathrm{eV}$ [44], leads to a photoelectron spectrum characterized by an easily observed, single vibrational progression with an energy separation of $302 \mathrm{meV}$ [45]. In our non-coincident TOF spectrum of Fig. 4, the first two vibrational states are readily separated. To single out the $v^{\prime}=2$ state is difficult because of the background from fast electrons in this type of spectrum. A least squares fit of PCI (Post Collision Interaction) profiles [45][46] to the CO C 1s spectrum in panel 4b) results in a Gaussian (apparatus) contribution to the linewidth of approx. $100 \mathrm{meV}$. (A fixed natural linewidth of $95 \mathrm{meV}$ was assumed [47].) Deconvolution of the monochromator bandpass [23] then results in a spectrometer resolution of ca. $50 \mathrm{meV}$ only. This resolution can, however, only be achieved in an experiment where the BESSY bunch clock is used as the stop signal. For coincidence data acquisition, with the Auger arrival times serving as a stop signal, an additional apparatus broadening for the photoelectron arises, which for the data shown amounts to about $150 \mathrm{meV}$. These contributions recombined are in reasonable agreement with the total photoelectron linewidth of $200 \mathrm{meV}$ quoted in [23]. 
Due to the higher photon energy and slightly less stringent retardation voltages applied, our new data on $\mathrm{O}_{2} \mathrm{O}$ 1s photoionization and subsequent KVV Auger decay reported below, as well as in Section IV, show a poorer energy resolution of $260 \mathrm{meV}$ for the non-coincident time-of-flight data and 350 meV total photoelectron energy resolution for the coincidence data. Data were recorded in $69000 \mathrm{~s}$ of subsequent acquisition time. Non-coincident count rates were approx. $800 \mathrm{~Hz}$ for the Auger spectrum and $60000 \mathrm{~Hz}$ in all TOF analysers added up. Approximately 47000 true coincident events have been detected, which corresponds to a rate of true coincidences of about $0.7 \mathrm{~Hz}$.

In Fig. 6 we present a plot of the arrival times $\tau_{\text {au,rel }}$ of $n o n$-coincident KVV Auger electrons from $\mathrm{O}_{2}$ (a part of the spectrum), referred to the BESSY bunch clock. The zero of $\tau_{\text {au,rel }}$ has been arbitrarily placed at the first hybrid bunch. (Absolute times-of-flight in the hemispherical analyser cannot be determined in this experiment, but were discussed earlier [26][42].) The vertical coordinate of the colour-coded map is the electron kinetic energy $\varepsilon_{\text {au, }}$ determined from the arrival position on the detector anode as discussed below. While the top panel of the figure gives the $\varepsilon_{\mathrm{au}} \mathrm{vs.} \tau_{\mathrm{au}, \mathrm{rel}}$ relationship for all 20 million non-coincident Auger electrons recorded, in the main panels we focus on the electrons received from one of the hybrid bunches. From a horizontal cut through the two-dimensional map in the Fig. 6 bottom panel we can determine the TOF spread in the Scienta analyser for a bundle of monoenergetic electrons as $6.2 \mathrm{~ns}$ (FWHM). This figure is the additional uncertainty in the determination of the photoelectron time-of-flight, $\tau_{\mathrm{ph}}$, which is encountered when we use the Auger electron arrival time as the point of reference for the $\tau_{\mathrm{ph}}$ measurement. Comparing to the simulations in Fig. 3 we see that the latter is comparable, but not larger than the spread in $\tau_{\mathrm{ph}}$ due to source volume and solid angle effects. The spread in $\tau_{\text {au,rel }}$ discussed above should not be confused with the $\tau_{\text {au,rel }}$ vs. $\varepsilon_{\mathrm{au}}$ dispersion, which is seen as the slope of the hybrid bunch Auger line in Fig. 6. This latter effect is corrected mathematically in our data analysis procedure. Both the spread in $\tau_{\mathrm{au}, \text { rel }}$ as well as the dependence of $\tau_{\mathrm{au}, \mathrm{rel}}$ on $\varepsilon_{\mathrm{au}}$ arise from different lengths of the trajectories through the analyser hemispheres [26]. Similar data for a smaller analyser have been presented by Gießel et al. [43] 
A core level photoelectron TOF spectrum, referred to the Auger arrival time, is shown in Fig. 7. Due to the operation in multi-bunch mode, most coincident electron pairs detected are accidental, with the two electrons resulting from two different SR bunches. True coincidences pertaining to the two exchange split peaks of the O 1s main line [45] are in the centre of the figure. Below the line of true + random coincidences we show an estimate of the random contribution to the signal (dots). This we calculate from event pairs, in which the two electrons were produced within different revolution periods of BESSY.

The details of Fig. 7 depend on the settings of the data acquisition hardware. In our experiments, the BESSY bunch marker signal was used as 'start' for a time-of-flight measurement. Events were then collected for a period of two full revolutions of the electron beam in the storage ring (approx. 1600 ns). This time is dictated by hardware limitations. If any events were detected, they were then processed by the TDC firmware while a new measurement cycle was initiated. Thus the measurement process is practically dead-time free. The true coincidences recorded such appear at small values of the arrival time difference (independent axis in Fig. 7). The accidental coincidences ideally form a smooth triangle with a baseline length of twice the revolution period. Its remnant structure results from the time structure of the BESSY fill pattern. Detailed discussion of the true and accidental coincidence spectra have been given for similar acquisition schemes [48][49]. Two facts we have experienced in our work are corroborated in [49]: (i). The net acquisition time cannot be reduced by increasing the SR flux, or the target density, since the accidental coincidence rate scales quadratically with these factors, while the true coincidence rate has a linear dependence. The true-to-random ratio therefore becomes worse, such that the statistical quality of the data recorded in a certain total time $T$ is invariant. A reasonable trade-off between count rate and true-to-random ratio $\left(R_{\mathrm{T}} / R_{\mathrm{Acc}}\right)$ for the experiments shown here was found at $R_{\mathrm{T}} / R_{\mathrm{Acc}}$ of $0.5-2$. (ii). Performing our experiment in multi-bunch as opposed to single-bunch data acquisition reduces the random coincidence count rate perceived for a given rate of true coincidences by a factor of $\tau_{\mathrm{bb}} /\left(\tau_{\mathrm{e}} F\right)=800 / 20 * 350 / 400=35$, where $\tau_{\mathrm{bb}}$ is the single bunch period, $\tau_{\mathrm{e}}$ the width of the photoelectron part of the TOF spectrum, and $F$ a factor which corrects for 
the incomplete filling of the ring in multibunch operation. $F$ can be written as the number of electron bunches in a completely filled ring, divided by the actual number of electron bunches.

\section{Data analysis}

Due to the use of a position-sensitive anode in our hemispherical analyser, a band of energies centred around the pass energy (the energy of the central, concentric trajectory) can be monitored simultaneously. Its full width is roughly $10 \%$ of the pass energy. For calibration purposes the Ar $\mathrm{L}_{2,3} \mathrm{M}_{2,3} \mathrm{M}_{2,3}$ Auger spectrum was recorded with the same settings as the $\mathrm{O}_{2}$ Auger spectrum, fitted by a least squares program, and compared to literature data [50]. We have thus been able to correct small non-linearities of the Scienta energy axis and have determined the energy resolution as 182(10) meV under the conditions used (pass energy $75 \mathrm{eV}, 500 \mu \mathrm{m}$ curved slit). The relative peak areas of the spectrum recorded on the analyser MCP were found to agree with Ref. [50] within $6 \%$. Therefore, no correction for differing transmission functions for the electrons within the analyser window was made.

For the photoelectron spectra, a time-to-energy $(t-E)$ conversion is necessary. The approach used in our work is described in Ref. [29][51]. Briefly, a ( $t-E)$ conversion function is determined from calibration data consisting of a set of line positions with known kinetic energies. An equation based on the non-relativistic expression for the electron kinetic energy is used. However, the physical dimensions of the analyser, the TDC channel pertaining to a vanishing time-of-flight, and the effective value of the retardation voltage averaged over the flight path are treated as fitting parameters to reproduce best the calibration data. Experimentally, these data were determined as peak centers of an isolated photoline excited by the hybrid bunch. It is desirable to perform the time-to-energy conversion such that the total area of the spectra is conserved in a mathematical sense. That is, the integral over the transformed spectrum $\tilde{f}(E)$ should equal the integral over the channel spectrum $f(t)$. This necessitates multiplication of the transformed data by a Jacobian factor $j:=d t / d E$,

since $\int \mathrm{d} t f(t)=\int \mathrm{d} E f(t(E))(d t / d E)$. We calculate $j$ from our $t$ - $E$ relationship, multiply all points of the original data set by it and resample the resulting function $\tilde{f}=f j$ on a grid which is 
equally spaced in energy. As the width of a TDC bin is much lower than the effective time resolution, the original spectrum $f(t)$ typically contains much more data points than are meaningful for $\tilde{f}(E)$. The width of the energy grid is therefore chosen such that it implies an averaging over several time channels.

\section{E. Comparison with other systems}

To close this Section we briefly compare our set-up with other systems for electron-electron coincidence spectroscopy which have been described in the literature. We restrict this discussion to instruments that have been used for synchrotron radiation studies, since a complete review of the subject including $(e, 2 e)$ experiments is outside the scope of this article. The set-ups used with SR excitation can be grouped in three categories: 1. Time-of-flight spectrometers, 2. Conventional dispersive instruments, 3. Projecting spectrometers, aka. reaction microscopes. Linear time-of-flight spectrometers have been used for atomic and molecular coincidence studies [15][31][52]. They combine good to moderate energy resolution with the capability to record a band of energies in parallel. Their prime use is therefore in the investigation of simultaneous photo-doubleionisation, where both ejected electrons can share the excess energy arbitrarily. For molecular photoelectron - Auger electron coincidences (CO C 1s, K-VV), an estimated resolution of $200 \mathrm{meV}$ for the photoelectron and $500 \mathrm{meV}$ for the Auger electron has been achieved [53][54]. Experiments so far were conducted such that all electron flight times were shorter than the synchrotron radiation bunch period, which necessitates use of a single or few bunch mode. The acceptance angle of such analysers is small, comparable to hemispherical electron analysers at best. This can be overcome by using an inhomogeneous magnetic field to guide the electrons first into a linear drift tube, and after some flight path onto a detector ('magnetic bottle spectrometer' [55]). Application of this principle for photoelectron, Auger electron coincidence studies has been described [56]. The solid angle accepted by the spectrometer can be greatly enhanced, up to nearly $4 \pi \mathrm{sR}$ [57]. This is at the cost of energy resolution however. While in many electrostatic analysers and in linear TOF analysers electrons in the region of interest can be strongly retarded to maximise the energy resolution, for magnetic bottle type instruments this has not been possible to the same extent. A representative study for one instrument in 
Ref. [58] gives a $\Delta \mathrm{E} / \mathrm{E}$ of $1.6 \%$ for electrons above $1 \mathrm{eV}$ kinetic energy. Nevertheless, useful results in atomic [58][59] and molecular [57] Auger coincidence studies were obtained.

Various combinations of two electrostatic analysers for coincidence acquisition have been described [10][11][13][36][49][60][61][62]. Of these, however, only the instruments which employ hemispherical analysers seem to be capable to retard fast (several hundred eV of kinetic energy) electrons efficiently to a pass energy which allows high resolution detection [49][62]. The solid angle accepted is then small. A combination of a cylindrical mirror analyser with a linear TOF spectrometer has been described by Wehlitz et al. [63].

In synchrotron radiation experiments, the kinetic energy of the photoelectrons is at the disposition of the experimentalist. Some groups have chosen to deliberately detect threshold photoelectrons only, as this can easily be done with a high solid angle and good energy resolution, see e.g. Ref.s $[16][17][18][64][65][66]$.

Finally, projecting electron spectrometers employing electric fields only ('velocity map imaging spectrometers') have found their place in synchrotron radiation based electron spectroscopy, but are not capable of detecting coincident electron pairs resolved by energy, as the inversion of the projected spectrum into energy space cannot be performed on an event by event basis. This can be overcome by overlaying a magnetic field ('reaction microscope'). However, various trade-offs in practice have hitherto limited this technique to the low resolution detection of electrons below some tens of eV kinetic energy [67][68]. In addition, the analysis of data from these experiments is non-trivial. An interesting novel development is to retrieve the Auger electron energy from a measurement of the recoil momentum imparted to the ion(s). Thus, the measurement of angular correlations between photo- and Auger electrons has recently become possible [69][70].

\section{Molecular photoelectron-Auger electron coincidence spectra: typical features}

Here we highlight some general features of molecular photoelectron-Auger electron coincidence spectra using the simulated spectrum of Fig. 1. Details of the figure are as follows: An inner shell photoelectron with a nominal adiabatic kinetic energy of $10 \mathrm{eV}$ is emitted, followed by a subsequent 
KVV Auger decay of the core hole at a nominal kinetic energy of $480 \mathrm{eV}$, and a lifetime broadening of $150 \mathrm{meV}$. Two vibrationally excited core-hole states, with a (harmonic) vibrational quantum energy of $\hbar \omega=200 \mathrm{meV}$, and intensities according to a Poisson distribution with a parameter $\left(I\left(v^{\prime}=1\right) / I\left(v^{\prime}=0\right)\right)$ of 0.8 [45] are seen at lower kinetic energy. The same nominal Auger energy is assumed for all vibrational states, which is the case when the potential curves of the singly and doubly excited states are parallel (within the range of nuclear coordinates which can be accessed by the intermediate state). Apparatus broadening effects are modelled by convolution with Gaussian profiles, the width (FWHM) of which were set to $50 \mathrm{meV}$ (photon bandpass) and $80 \mathrm{meV}$ (photoelectron and Auger electron analyser). Intensities of the colour-coded map are given as counts/eV ${ }^{2}$, and of the non-coincident plots as counts/eV. Integration of these in a mathematical sense leads to an assumed intensity of approx. 100,000 coincidence counts. The Coulomb interaction of the two electrons in the continuum (postcollision interaction, PCI) is taken into account [46].

A considerable broadening of the profiles along the lines of constant total energy is seen. These are diagonals with a negative slope in the central panel. The projection of this broadening onto either the photoelectron or Auger electron kinetic energy axis leads to the well-known Lorentzian profiles. Perpendicular to these diagonals the features of the coincident electron spectrum are broadened only by the apparatus contributions (dotted lines in Fig. 1). If the latter can be made smaller than the lifetime broadening, the opportunity to observe coincident spectral features with a sub-natural resolution is evident.

Spectra obtained with our apparatus have, after time-to-energy conversion of the photoelectron, a similar structure. One sees that by resolving the energies of each photoelectron-Auger electron pair, the transitions for a molecule can be decomposed into contributions from different vibrational states. The Auger energy obviously depends on the combination of $v^{\prime}$ and $v^{\prime \prime}$, the intermediate and final state vibrational quantum numbers. Which combinations of $v^{\prime}, v^{\prime \prime}$ are effective in the most simple case is given by the Franck-Condon (FC) principle, applied to the intermediate and final state potential curves. More generally, different electronic states may also contribute to overlapping lines in a noncoincident Auger spectrum that can be resolved by a coincidence measurement. Potential curves for 
the intermediate state can be bound or dissociative [45], while for the potential curve of the final state asymptotic dissociation is expected, as a small dication cannot be stable. It has been found, however, that the energetically lowest dicationic states of numerous small molecules are metastable in the sense that they support a local minimum of the potential curve, with a potential well to the dissociative branch at larger values of some internuclear coordinate [71]. But, even if FC transitions between discrete vibrational states provide an appropriate description, they are too numerous in most cases to be disentangled in the non-coincident Auger spectrum (although meticulous lineshape analysis of high quality non-coincident data has led to a success for some systems [72]). The simulated spectrum in Fig. 1 shows a case with parallel potential curves of intermediate and final state, under which conditions Auger transitions between identical values of $v^{\prime}, v^{\prime \prime}$ are strongly favoured.

Using a coincident technique a more detailed picture of these Auger transitions is revealed. This is achieved by plotting the experimental transition intensity not vs. Auger kinetic energy $\varepsilon_{\mathrm{au}}$, but vs. final state energy $E_{\mathrm{fi}}$. As these two quantities are connected by $E_{\mathrm{fi}}=I P-\varepsilon_{\mathrm{au}}$, with $I P$ denoting the core level ionization potential, mathematically the conversion corresponds to a shear of the recorded coincidence map by $45^{\circ}$. Then, all transitions populating a certain final state line up along one of the coordinate axes (Fig. 8). This resembles experiments in molecular photo-double-ionization by electron-electron coincidence methods (e.g. [73]), but we see the population of one electronic final state over a wider range of its potential curve, because an intermediate state is involved in the transition. One point apparent from Fig. 8 is the non-trivial shape which the coincident intensity regions acquire due to the combined effect of the finite electron analyser resolution, monochromator bandwidth and the shear transformation [23].

The lifetime of Auger decay and a molecular vibrational period are of the same order of magnitude. One therefore expects an influence of the molecular dynamics during the decay on the radiationless de-excitation spectrum. In a time-independent picture a simple model of such effects is known as lifetime vibrational interference (LVI) [20][74]; there is also some experimental evidence for this effect in normal Auger spectra [72]. With the new set-up we expect to be able to isolate the effect of LVI in normal Auger spectra better than is currently possible. 


\section{The photoelectron-Auger electron coincidence spectrum of molecular oxygen: Results and discussion}

As an instructive example, we report here new data for oxygen $\left(\mathrm{O}_{2}\right)$. The non-coincident photoelectron and Auger spectra both have been reported a number of times (e.g. [2]). For the O 1s spectrum, most recent data are by Sorensen et al. [75]. Earlier work has been reviewed in Ref. [45]. The spectrum is characterized by the exchange splitting between the ${ }^{2} \Sigma$ and ${ }^{4} \Sigma$ states of the cation, which amounts to $1.06 \mathrm{eV}$. This splitting is due to the spin coupling of the core hole with the triplet spin of the open, outermost valence shell of $\mathrm{O}_{2}$. The two resulting photoelectron lines differ in width, with the ${ }^{2} \Sigma$ line about 1.5 times as wide as the ${ }^{4} \Sigma$ line. This can be rationalized by the smaller influence of relaxation in the latter state. The exact decomposition of the observed profiles into vibrational contributions and a $g / u$ splitting of the core hole (26 meV in theory [76]) is as yet unknown. The KVV Auger spectrum has been discussed in detail by Sambe and Ramaker [77], Larsson et al. [78] and most recently by Bao et al. [79]. The dicationic states of the $\mathrm{O}_{2}$ molecule (and of a molecule in general) can also be accessed by direct photo-double-ionisation with detection of both photoelectrons. This method has proven to be useful for characterizing the energies and vibrational progressions of the doubly charged states in numerous species, and has been applied to $\mathrm{O}_{2}$ by Dawber et al. and by Eland [80][73].

In the following we will present some of our experimental results. By our data acquisition scheme, coincident and non-coincident spectra are recorded simultaneously. We present the conventional, noncoincident KVV Auger spectrum in the right hand side panel of Fig. 6. Compared to earlier work the structures are very similar [77][78] and the experimental resolution equals the one of Larsson et al. [78].

The Auger spectrum acquired in coincidence with the core level electrons is shown in Fig. 9. A transformation of the coincidence data into an intermediate state binding energy vs. final state energy representation has been carried out as discussed above. Here, we present the binding energy region of the lowest three dicationic states which are populated by Auger decay. Statistical errors of the data and the random coincidence background were propagated throughout the analysis procedure. The relative error of the intensity represented by a single pixel in the central panel of Fig. 9 lies between 10 and 30 
$\%$, for pixels exceeding 10000 counts/eV². Error bars on the one-dimensional sums are shown in the Figure. The subtraction of random coincidences leads to some noise which takes the appearance of diagonal trails in Fig. 9.

The lowest dicationic states seen in our experiment have been assigned as $\mathrm{W}^{3} \Delta_{\mathrm{u}}, \mathrm{B}^{3} \Pi_{\mathrm{g}}$ and $\mathrm{B}{ }^{3} \Sigma_{\mathrm{u}}^{-}$, while two energetically lower states, which have been detected in a zero-kinetic-energy electronelectron coincidence measurement [80], hardly receive intensity by Auger decay. The assignment is taken from earlier work [77][79]. The population of three final states can be easily seen in the summed spectrum. While two of them obviously receive intensity from both the ${ }^{2} \Sigma$ and the ${ }^{4} \Sigma$ intermediate states, our coincidence map suggests that the middle one, assigned to $\mathrm{B}^{3} \Pi_{\mathrm{g}}$, can be populated only from the ${ }^{4} \Sigma$ state. The Auger spectra separated with respect to the core-ionized state are presented again in Fig. 10, where the intensity for intermediate states in the binding energy intervals [543.1, 543.7] and [544.2, 544.7] eV has been summed up.

In a recent work by Bao et al. [79] separate Auger spectra of the ${ }^{2} \Sigma$ and ${ }^{4} \Sigma$ intermediate states were calculated. These results can be compared to our data in Fig. 10. We can distinguish transitions into final states with energies of 43.0, 43.7 and $44.1 \mathrm{eV}$. If we first compare the relative intensities to the calculations, we find significant differences for the middle one of the final states, the $\mathrm{B}^{3} \Pi_{\mathrm{g}}$. Experimentally we see a vanishing or very small intensity for the decay of the ${ }^{2} \Sigma$ intermediate into this state. In contrast, in [79] the three states theoretically received very similar intensities in decays from both intermediate states. Moreover the $\mathrm{B}^{3} \Pi_{\mathrm{g}}$ and $\mathrm{B}^{3} \Sigma_{\mathrm{u}}^{-}$states came out with the opposite order in energy compared to Fig. 10. This demonstrates that our experiment is indeed capable to give new information on the dynamics of molecular Auger decay. (In fact, the authors of [79] suggested corrections of their $a b$ initio calculations on the basis of peak-fitting a sum of their spectra to a conventional Auger spectrum. The empirical corrections given by this approach would lead to a better agreement of the calculations with our spectra.)

Compared to data from photo-double-ionization we mainly see that a wider range of vibrational states is populated at least for some states. The $\mathrm{B}^{3} \Pi_{\mathrm{g}}$ appears as just one single vibrational state in the photodouble-ionization spectrum both at threshold [80] and above [73]. On the other hand, the 
${ }^{1} \Sigma_{\mathrm{g}}^{+}$dicationic ground state appears in the photo-double-ionization spectrum, but not in the Auger spectrum. This can be rationalized from a propensity of the valence electrons participating in Auger decay to be singlet-coupled [79].

\section{Summary}

In summary, we have described an apparatus for photoelectron-Auger electron coincidence spectroscopy which combines several electron time-of-flight spectrometers with a large hemispherical electron analyser. We have described and discussed the general features of this form of electron spectroscopy applied to free molecules and clusters and, in particular, we illustrate its potential for the investigation of molecular Auger decay with high resolution. Moreover, we show the first data for the diatomics $\mathrm{CO}$ [23] and $\mathrm{O}_{2}$ (hitherto unpublished). The latter are compared to existing calculations.

\section{Acknowledgements}

UH would like to thank Jens Viefhaus and Georg Prümper for valuable discussions on various aspects of this project. This work was partially funded by the Deutsche Forschungsgemeinschaft (He 3060/4) and the Fonds der chemischen Industrie. The expertise of BESSY in running the storage ring and maintaining the beamlines is gratefully acknowledged. 


\section{References}

[1] R.E. Weber, W.T. Peria, J. Appl. Phys. 38 (1967) 4355.

[2] K. Siegbahn, C. Nordling, G. Johansson, J. Hedman, P.F. Héden, K. Hamrin, U. Gelius, T. Bergmark, L.O. Werme, R. Manne, Y. Baer: ESCA applied to free molecules, North-Holland, Amsterdam, 1969.

[3] F.P. Larkins, J. Electron Spectrosc. Relat. Phenom. 51 (1990) 115.

[4] A. Cesar, H. Ågren, V. Carravetta, Phys. Rev. A 40 (1989) 187.

[5] L. S. Cederbaum and F. Tarantelli, J. Chem. Phys. 98 (1993) 9691; J. Chem. Phys. 99 (1993) 5871.

[6] F. Tarantelli and L. S. Cederbaum, Phys. Rev. Lett. 71 (1993) 649.

[7] T. X. Carroll, J. Hahne, T. D. Thomas, L. J. Sæthre, N. Berrah, J. Bozek, E. Kukk, Phys. Rev. A 61 (2000) 042503 and references therein.

[8] T. D. Thomas, C. Miron, K. Wiesner, P. Morin, T. X. Carroll, L. J. Sæthre, Phys. Rev. Lett. 89 (2002) 223001.

[9] H. W. Haak, G. A. Sawatzky, T. D. Thomas, Phys. Rev. Lett. 41 (1978) 1825.

[10] E. Jensen, R. A. Bartynski, S. L. Hulbert, E. D. Johnson, R. Garrett, Phys. Rev. Lett. 62 (1989) 71.

[11] G. Stefani, R. Gotter, A. Ruocco, F. Offi, F. Da Pieve, S. Iacobucci, A. Morgante, A. Verdini, A. Liscio, H. Yao, R. A. Bartynski, J. Electron Spectrosc. Relat. Phenom. 141 (2004) 149.

[12] B. Kämmerling, V. Schmidt, Phys. Rev. Lett. 67 (1991) 1848.

[13] V. Schmidt: Electron Spectrometry of Atoms using Synchrotron Radiation, University Press, Cambridge, 1997.

[14] M. Neeb, J.-E. Rubensson, M. Biermann, W. Eberhardt, J. Phys. B 29 (1996) 4381.

[15] J. Viefhaus, G. Snell, R. Hentges, M. Wiedenhöft, F. Heiser, O. Gessner, U. Becker, Phys. Rev. Lett. 80 (1998) 1618.

[16] P. Lablanquie, F. Penent, R.I. Hall, H. Kjeldsen, J.H.D. Eland, A. Muehleisen, P.

Pelicon, Z. Šmit, M. Zitnik, F. Koike, Phys. Rev. Lett. 84 (2000) 47.

[17] Y. Hikosaka, P. Lablanquie, F. Penent, J.G. Lambourne, R.I. Hall, T. Aoto, K. Ito, J. Electron Spectrosc. Relat. Phenom. 137-140 (2004) 287.

[18] Y. Hikosaka, P. Lablanquie, E. Shigemasa, T. Aoto, K. Ito, J. Phys. B 41 (2008) 025103.

[19] E. Pahl, H.-D. Meyer, L. S. Cederbaum, Z. Phys. D 38 (1996) 215; Z. W. Gortel, R. Teshima, D. Menzel, Phys. Rev. A 58 (1998) 1225.

[20] F. Gelmukhanov and H. Ågren, Phys. Rep. 312 (1999) 87. 
[21] A. Kivimäki, A. Naves de Brito, S. Aksela, H. Aksela, O.-P. Sairanen, A. Ausmees, S. J. Osborne, L. B. Dantas, S. Svensson, Phys. Rev. Lett. 71 (1993) 4307.

[22] A. Kivimäki, K. Maier, U. Hergenhahn, M. N. Piancastelli, B. Kempgens, A. Rüdel, A. M. Bradshaw, Phys. Rev. Lett. 81 (1998) 301.

[23] V. Ulrich, S. Barth, S. Joshi, T. Lischke, A. M. Bradshaw, U. Hergenhahn, Phys. Rev. Lett. 100 (2008) 143003.

[24] S. P. Marburger, O. Kugeler, U. Hergenhahn, in Synchrotron Radiation Instrumentation: Eighth International Conference, edited by T. Warwick, J. Arthur, H. A. Padmore and J. Stöhr (American Institute of Physics, San Francisco, 2003) Vol. 705, p. 1114. [25] N. Mårtensson, P. Baltzer, P. A. Brühwiler, J.-O. Forsell, A. Nilsson, A. Stenborg, B. Wannberg, J. Electron Spectrosc. Relat. Phenom. 70 (1994) 117.

[26] O. Kugeler, S. Marburger, U. Hergenhahn, Rev. Sci. Instrum. 74 (2003) 3955.

[27] F. Senf, F. Eggenstein, R. Follath, S. Hartlaub, H. Lammert, T. Noll, J. S. Schmidt, G. Reichardt, O. Schwarzkopf, M. Weiss, T. Zeschke, W. Gudat, Nucl. Instrum. Methods A 467468 (2001) 474.

[28] J. Bahrdt, W. Frentrup, A. Gaupp, M. Scheer, W. Gudat, G. Ingold, S. Sasaki, Nucl. Instrum. Methods A 467-468 (2001) 21.

[29] O. Hemmers, S. B. Whitfield, P. Glans, H. Wang, D. W. Lindle, R. Wehlitz, I. A. Sellin, Rev. Sci. Instrum. 69 (1998) 3809.

[30] P. Feulner, P. Averkamp, B. Kassühlke, Appl. Phys. A 67 (1998) 657.

[31] U. Becker, J. Electron Spectrosc. Relat. Phenom. 112 (2000) 47.

[32] U. Becker and B. Langer, Nucl. Instrum. Methods A 601 (2009) 78.

[33] G. Snell, J. Viefhaus, F. B. Dunning, N. Berrah, Rev. Sci. Instrum. 71 (2000) 2608.

[34] S. J. Buckman, R. J. Gulley, M. Moghbelalhossein, S. J. Bennett, Meas. Sci. Technol. 4 (1993) 1143.

[35] B. Kämmerling, V. Schmidt, J. Phys. B 26 (1993) 1141.

[36] P. Bolognesi, A. De Fanis, M. Coreno, L. Avaldi, Phys. Rev. A 70, 022701 (2004).

[37] K. Godehusen, H.-C. Mertins, T. Richter, P. Zimmermann, M. Martins, Phys. Rev. A 68 (2003) 012711.

[38] P. Bolognesi, D. Toffoli, P. Decleva, V. Feyer, L. Pravica, L. Avaldi, J. Phys. B 41 (2008) 221002, and references therein.

[39] K. Hosaka, J. Adachi, A.V. Golovin, M. Takahashi, T. Teramoto, N. Watanabe, T. Jahnke, T. Weber, M. Schöffler, L. Schmidt, T. Osipov, O. Jagutzki, A.L. Landers, M.H. Prior, H. Schmidt-Böcking, R. Dörner, A. Yagishita, S.K. Semenov, N.A. Cherepkov, Phys. Rev. A 73 (2006) 022716. 
M. Abo-Bakr, W. Anders, P. Kuske, G. Wüstefeld, in Particle Accelerator Conference, edited by P. L. Joe Chew and Sara Webber (IEEE, Portland, OR, 2003) Vol. Catalog Number 03CH37423C, p. 3020.

[41] K. Holldack, M. v. Hartrott, F. Hoeft, O. Neitzke, E. Bauch, M. Wahl, in Advanced Photon Counting Techniques II, edited by W. Becker (SPIE, 2007) Vol. 6771, p. 677118.

[42] O. Kugeler, PhD thesis, Technische Universität Berlin, 2003.

[43] T. Gießel, D. Bröcker, P. Schmidt, W. Widdra, Rev. Sci. Instrum. 74 (2003) 4620.

[44] V. Myrseth, J. D. Bozek, E. Kukk, L. J. Sæthre, T. D. Thomas, J. Electron Spectrosc. Relat. Phenom. 122 (2002) 57.

[45] U. Hergenhahn, J. Phys. B 37 (2004) R89.

[46] M. Y. Kuchiev and S. A. Sheinerman, Soviet Physics-JETP 63 (1986) 986.

[47] T. X. Carroll, K. J. Børve, L. J. Sæthre, J. D. Bozek, E. Kukk, J. A. Hahne, T. D. Thomas, J. Chem. Phys. 116 (2002) 10221.

[48] E. Jensen, R. A. Bartynski, S. L. Hulbert, E. D. Johnson, Rev. Sci. Instrum. 63 (1992) 3013.

[49] P. Calicchia, S. Lagomarsino, F. Scarinci, C. Martinelli, V. Formoso, Rev. Sci. Instrum. 70 (1999) 3529.

[50] H. Pulkkinen, S. Aksela, O.-P. Sairanen, A. Hiltunen, H. Aksela, J. Phys. B 29 (1996) 3033.

[51] Note that the sentence below equation (3) of [29] should read: 'The next step is to linearize this data set by plotting $1 /(\text { prompt-ch) })^{2}$ with respect to the kinetic energy...'

[52] J. Viefhaus, S. Cvejanovic, B. Langer, T. Lischke, G. Prümper, D. Rolles, A. V. Golovin, A. N. Grum-Grzhimailo, N. M. Kabachnik, U. Becker, Phys. Rev. Lett. 92 (2004) 083001.

[53] J. Viefhaus, U. Hergenhahn, R. Hentges, A. Rüdel, U. Becker, (Hasylab Annual Report, 1998).

[54] J. Viefhaus, U. Hergenhahn, R. Hentges, A. Rüdel, U. Becker in ICPEAC XXI, Abstr. of Contrib. Papers, ed. by Y. Itikawa, reprinted in [31].

[55] P. Kruit and F. H. Read, J. Phys. E 16 (1983) 313.

[56] F. Penent, P. Lablanquie, R.I. Hall, J. Palaudoux, K. Ito, Y. Hikosaka, T. Aoto, J.H.D. Eland, J. Electron Spectrosc. Relat. Phenom. 144-147 (2005) 7.

[57] T. Kaneyasu, Y. Hikosaka, E. Shigemasa, P. Lablanquie, F. Penent, K. Ito, J. Phys. B 41 (2008) 135101.

[58] P. Lablanquie, L. Andric, J. Palaudoux, U. Becker, M. Braune, J. Viefhaus, J. H. D. Eland, F. Penent, J. Electron Spectrosc. Relat. Phenom. 156-158 (2007) 51.

[59] F. Penent, J. Palaudoux, P. Lablanquie, L. Andric, R. Feifel, J.H.D. Eland, Phys. Rev. Lett. 95 (2005) 083002. 
[60] T. J. Reddish, G. Richmond, G. W. Bagley, J. P. Wightman, S. Cvejanovic, Rev. Sci. Instrum. 68 (1997) 2685.

[61] R. Gotter, A. Ruocco, A. Morgante, D. Cvetko, L. Floreano, F. Tommasini, G. Stefani, Nucl. Instrum. Methods A 467-468 (2001) 1468.

[62] P. Bolognesi, R. Püttner, L. Avaldi, Chem. Phys. Lett. 464 (2008) 21.

[63] R. Wehlitz, L. S. Pibida, J. C. Levin, I. A. Sellin, Rev. Sci. Instrum. 70 (1999) 1978.

[64] K. Lee, S.L. Hulbert, P. Kuiper, D. Ji, D.M. Hanson, Nucl. Instrum. Methods A 347 (1994) 446.

[65] Y. Hikosaka, F. Penent, P. Lablanquie, R. Hall, K. Ito, Meas. Sci. Technol. 11 (2000) 1697.

[66] P. Lablanquie, S. Sheinerman, F. Penent, R.I. Hall, M. Ahmad, T. Aoto, Y. Hikosaka, K. Ito, J. Phys. B 35 (2002) 3265.

[67] R. Dörner, V. Mergel, O. Jagutzki, L. Spielberger, J. Ullrich, R. Moshammer, H. Schmidt-Böcking, Phys. Rep. 330 (2000) 95.

[68] J. Ullrich, R. Moshammer, A. Dorn, R. Dörner, L. P. H. Schmidt, H. SchmidtBöcking, Rep. Prog. Phys. 66 (2003) 1463.

[69] A.L. Landers, F. Robicheaux, T. Jahnke, M. Schöffler, T. Osipov, J. Titze, S.Y. Lee, H. Adaniya, M. Hertlein, P. Ranitovic, I. Bocharova, D. Akoury, A. Bhandary, T. Weber, M.H. Prior, C.L. Cocke, R. Dörner, A. Belkacem, Phys. Rev. Lett. 102 (2009) 223001.

[70] N.A. Cherepkov, S.K. Semenov, M.S. Schöffler, J. Titze, N. Petridis, T. Jahnke, K. Cole, L.P.H. Schmidt, A. Czasch, D. Akoury, O. Jagutzki, J.B. Williams, C.L. Cocke, T. Osipov, S. Lee, M.H. Prior, A. Belkacem, A.L. Landers, H. Schmidt-Böcking, T. Weber, R. Dörner, Phys. Rev. A 80 (2009) 051404.

[71] D. Schröder and H. Schwarz, J. Phys. Chem. A 103 (1999) 7385.

[72] R. Püttner, X.-J. Liu, H. Fukuzawa, T. Tanaka, M. Hoshino, H. Tanaka, J. Harries, Y. Tamenori, V. Carravetta, K. Ueda, Chem. Phys. Lett. 445 (2007) 6 and references therein.

[73] J. H. D. Eland, Chem. Phys. 294 (2003) 171.

[74] F. Kaspar, W. Domcke, L. S. Cederbaum, Chem. Phys. 44 (1979) 33; F. Gelmukhanov and H. Ågren, Phys. Rep. 312 (1999) 87.

[75] S. L. Sorensen, K. J. Børve, R. Feifel, A. de Fanis, K. Ueda, J. Phys. B 41 (2008) 095101.

[76] N. Kosugi, Chem. Phys. 289 (2003) 117.

[77] H. Sambe and D. E. Ramaker, Chem. Phys. 104 (1986) 331.

[78] M. Larsson, P. Baltzer, S. Svensson, B. Wannberg, N. Mårtensson, A. Naves de Brito, N. Correia, M. P. Keane, M. Carlsson-Göthe, L. Karlsson, J. Phys. B 23 (1990) 1175.

[79] Z. Bao, R. F. Fink, O. Travnikova, D. Céolin, S. Svensson, M. N. Piancastelli, J. Phys. B 41 (2008) 125101. 
[80] G. Dawber, A. G. McConkey, L. Avaldi, M. A. MacDonald, G. C. King, R. I. Hall, J. Phys. B 27 (1994) 2191. 


\section{Figure captions}

FIG. 1. Simulated sketch of a molecular photoelectron, Auger electron coincidence spectrum measured with high energy resolution. The colour-coded map shows the simulated intensity of electron pairs for the respective combinations of energies; the one-dimensional plots on the top and right-hand side show the coincident intensity integrated along one of the energy axes. These correspond to conventionally measured photoelectron and Auger electron spectra, respectively. Horizontal and vertical dotted lines indicate the full width at half maximum (FWHM) of the analyser bandpass used in the simulation, and diagonal dotted lines pertain to the photon bandpass. See text for details (beginning of Section III).

FIG. 2. Set-up for electron-electron coincidence measurements. The synchrotron radiation propagates towards the observer. The hemispherical detector is mounted at a downward angle of $54.7^{\circ}$ to the horizontal and within the plane perpendicular to the photon beam ('dipole plane'). The gas cell is mounted atop of an expansion chamber for cluster experiments (not drawn, see [24]). An array of six identical time-of-flight spectrometers is mounted in a vertical plane containing the radiation propagation axis, and at angles of $0^{\circ}, \pm 22.2^{\circ}, \pm 44.4^{\circ}$ and $66.6^{\circ}$ with respect to the vertical axis. (Positive sign = backward scattering direction.) Magnetic fields are actively compensated by a set of Helmholtz coils (not shown). An enlarged view of the interaction region is shown in the inset.

FIG. 3. Ray-tracing simulation of the time-of-flight (TOF) spectrometers with a flight path of $228.5 \mathrm{~mm}$ from the interaction region to the mesh. A total number of $2.7 \times 10^{5}$ (for the point source case, dotted curve) and $8.1 \times 10^{5}$ electrons (for the $44.4^{\circ}$ tilted line source, solid line) have been used to simulate the two spectra (see text for details).

FIG. 4. Electron spectra of CO excited with a photon energy of $304 \mathrm{eV}$, sum over all TOF analysers. The flight time in the TOF is referred to the BESSY bunch clock. The spectra are therefore a convolution of a TOF spectrum, including photo- and Auger electrons, with the time structure of the BESSY fill pattern (panel a). The vibrationally resolved, non-coincident CO C 1s photoelectron spectrum excited by the hybrid bunch can be recognized on-top of a background of fast electrons from 
other bunches. The $\mathrm{C} 1 \mathrm{~s}$ region is expanded in panel b. The first two vibrational substates of the $\mathrm{CO}^{+}$ $\mathrm{C} 1 \mathrm{~s}^{-1}$ cation, at an energy spacing of 302(3) $\mathrm{meV}$ [45], are clearly resolved. The step function seen in the intensity at around $\mathrm{t}=25 \mathrm{~ns}$ is caused by the dead-time of the acquisition electronics (Fig. 5).

FIG. 5. Schematic of the data acquisition electronics. The MCP pulses are amplified by pairs of commercial, inverting GHz amplifiers (Minicircuits), and further shaped by constant fraction discriminators (TOFs: Ortec, Scienta: Roentdek). A four channel multi-hit-capable time-to-digital converter (TDC) with 60 ps bin width and 16 bit signal depth collects the timing information for the norm pulses (GPTA, Berlin, Germany). The TDC start signal is provided by the BESSY bunch marker, a clock with the frequency of the inverse electron revolution time. All electron hits are used as stop signals, and, by the TDC firmware, are tagged with a bit pattern, which is incremented with the bunch marker pulses. For the hemispherical analyser, timing and energy information can be retrieved from the TDC signals as described in Ref. [26][42]. Coincident events involving detection of more than one electron can be separated in an off-line analysis of the data, and non-coincident data are extracted from the same set.

FIG. 6. Auger electron energy $\varepsilon_{\mathrm{au}}$ of electrons emitted by $\mathrm{O}_{2}$ molecules vs. arrival time $\tau_{\mathrm{au}, \mathrm{rel}}$ in the hemispherical analyser. The gross structure of the arrival time spectrum reflects the BESSY fill pattern [41], see panel a) and Fig. 4. A zoom into the Auger electrons received from the hybrid bunch (colour coded map in panel c) allows the TOF spread in the hemispherical analyser to be determined for monoenergetic electrons (panel b). For the panel b), electrons between the two dotted lines in panel c) have been taken into account. On the right-hand side (panel d) the non-coincident Auger spectrum (sum over all events shown in the panel a) is shown. The numbering of the lines follows Larsson et al. [78].

FIG. 7. Time-of-flight spectrum of $\mathrm{O}_{2} \mathrm{O}$ 1s photoelectrons detected in coincidence with K-VV Auger electrons. The flight time in the TOF is referred to the time of Auger electron detection. In the centre, the true coincidence signal with the two oxygen 1s main lines can be seen. Other events pertain to accidental coincidences, which have the shape of approximately a triangle in this plot. The line 
spectrum is the true + accidental signal; the points represent a spectrum of accidental coincidences only.

FIG. 8. Simulated sketch of a molecular photoelectron-Auger electron coincidence spectrum plotted as a function of intermediate $\left(E_{\mathrm{b}}\right)$ and final state $\left(E_{\mathrm{fi}}\right)$ binding energy. Same parameters as in Fig. 1 . See text for details.

FIG. 9. Experimental photoelectron-Auger electron coincidence spectrum of $\mathrm{O}$ 1s photoionization in $\mathrm{O}_{2}$, followed by K-VV Auger decay. The photon energy is $551.85 \mathrm{eV}$. The colour-coded map gives the intensity of electron pairs with the respective combinations of intermediate and final state binding energy. The top panel and the right-hand side panel contain the photoelectron intensity and the Auger electron intensity, respectively, summed over all energies of the other electron. Final state designations on the right-hand side follow the literature [79][73]. Intensities of the colour-coded map are given as counts/ $\mathrm{eV}^{2}$, and of the non-coincident plots as counts/eV.

FIG. 10. Experimental Auger spectra of the $\mathrm{O} 1 \mathrm{~s}^{-1}{ }^{4} \Sigma$ and the ${ }^{2} \Sigma$ core-ionized states, plotted as a function of final state energy. Error bars of characteristic size are shown for two data points. 


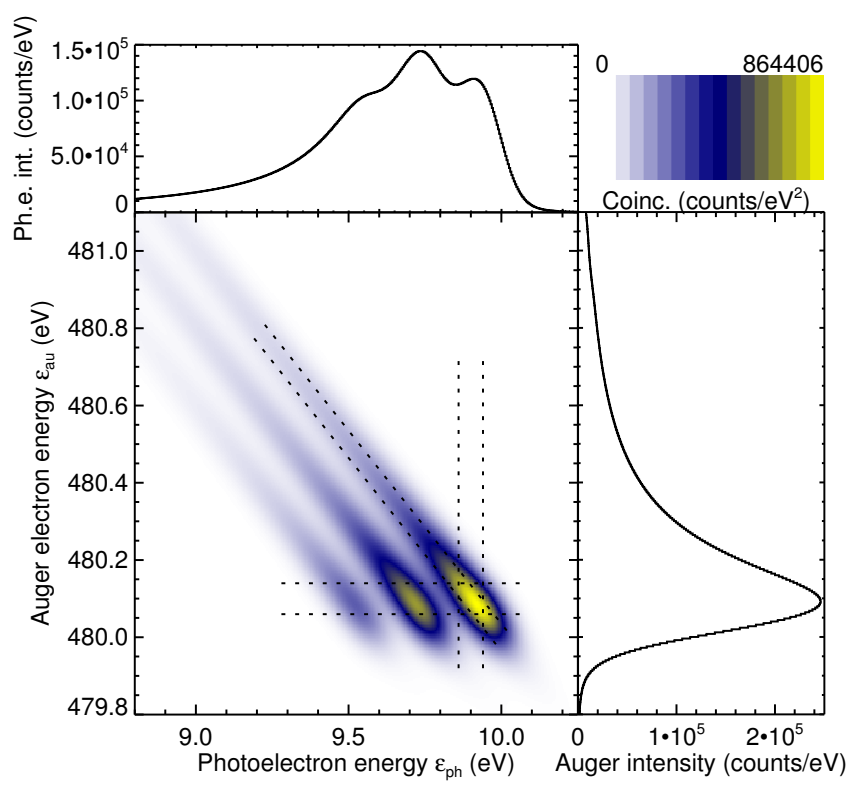

Fig. 1 


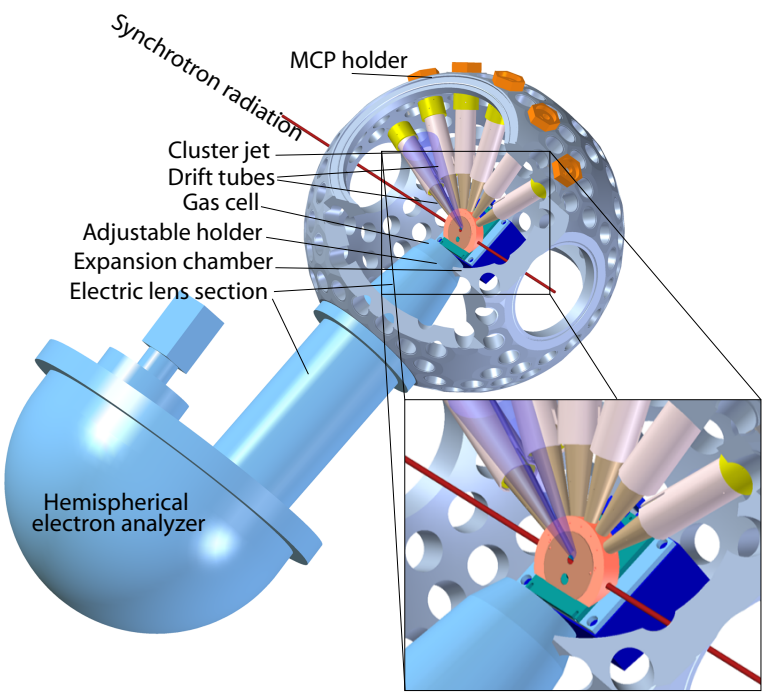

Fig. 2 


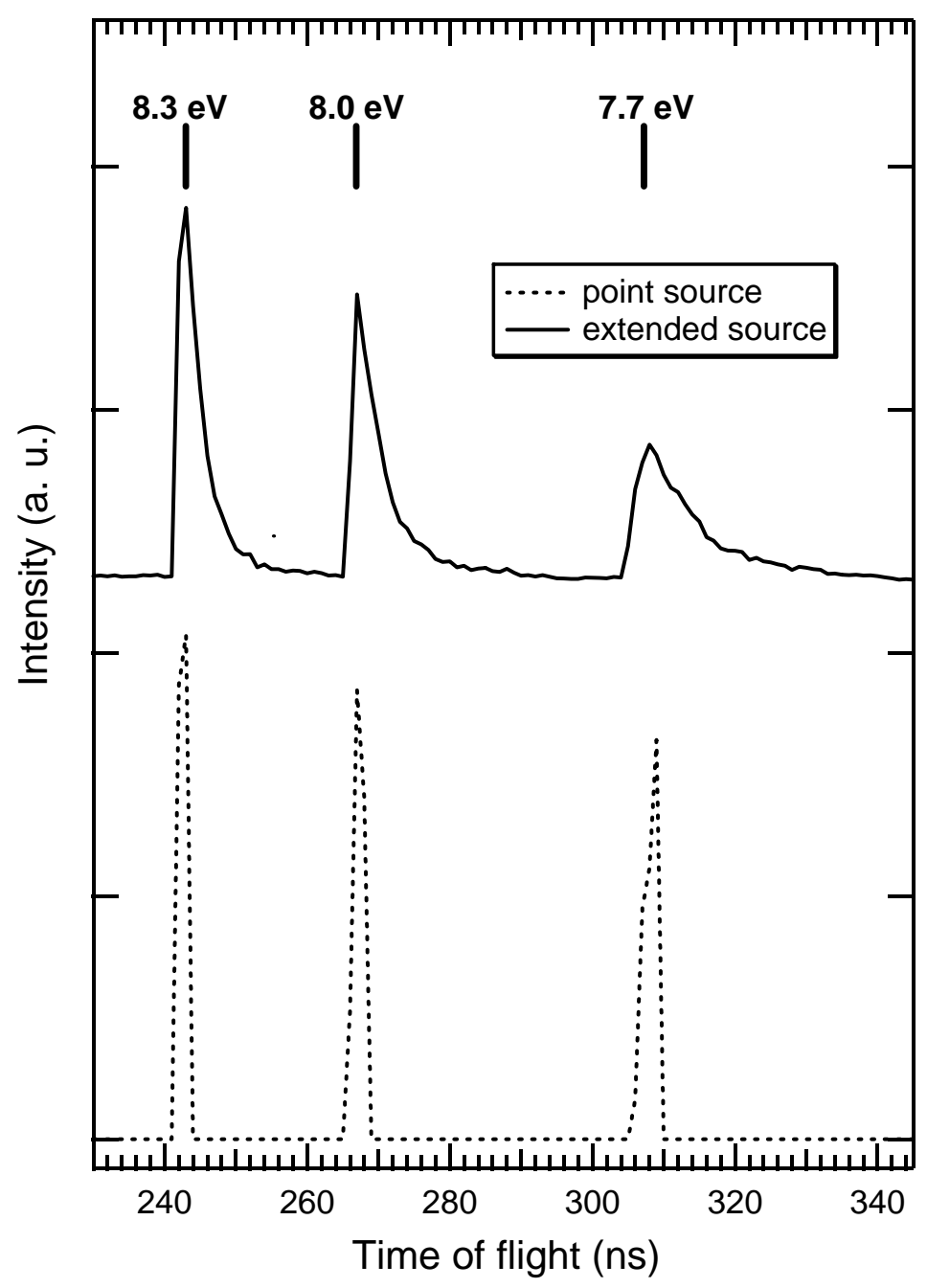

Fig. 3 


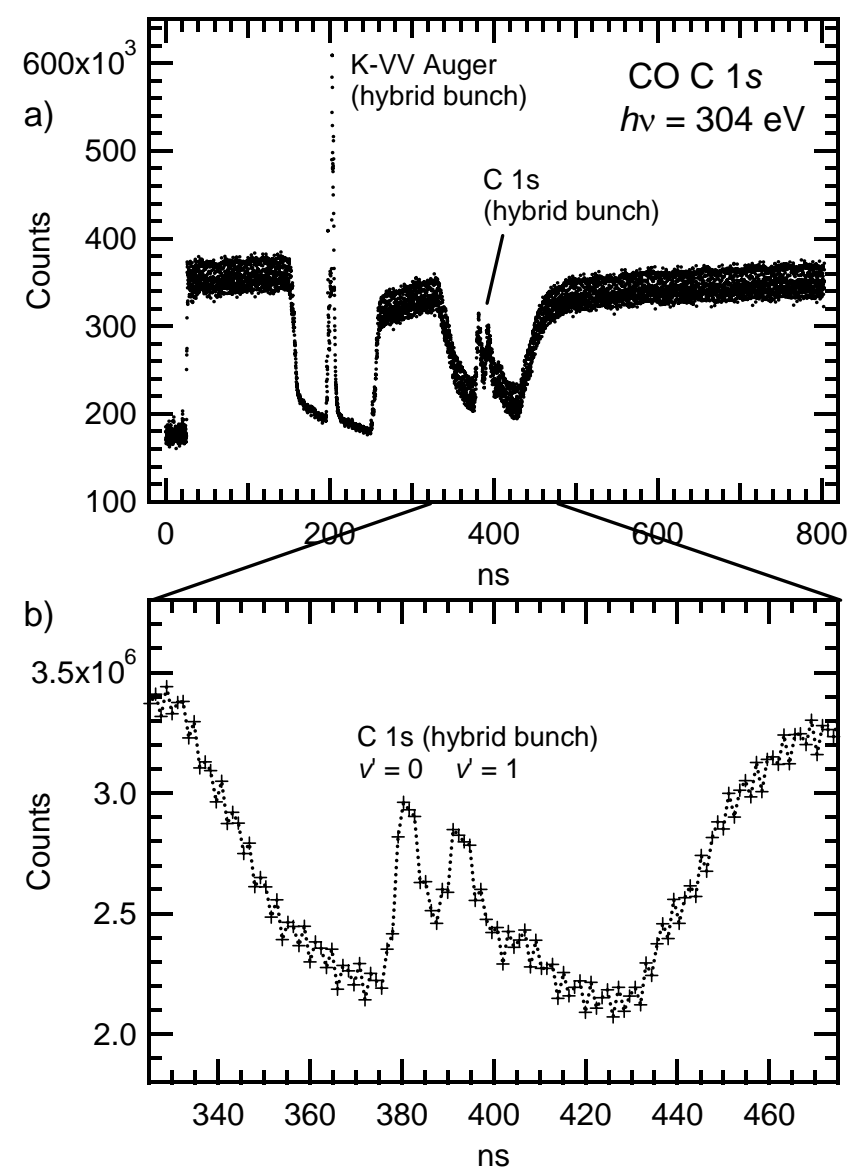

Fig. $\quad 4$ 


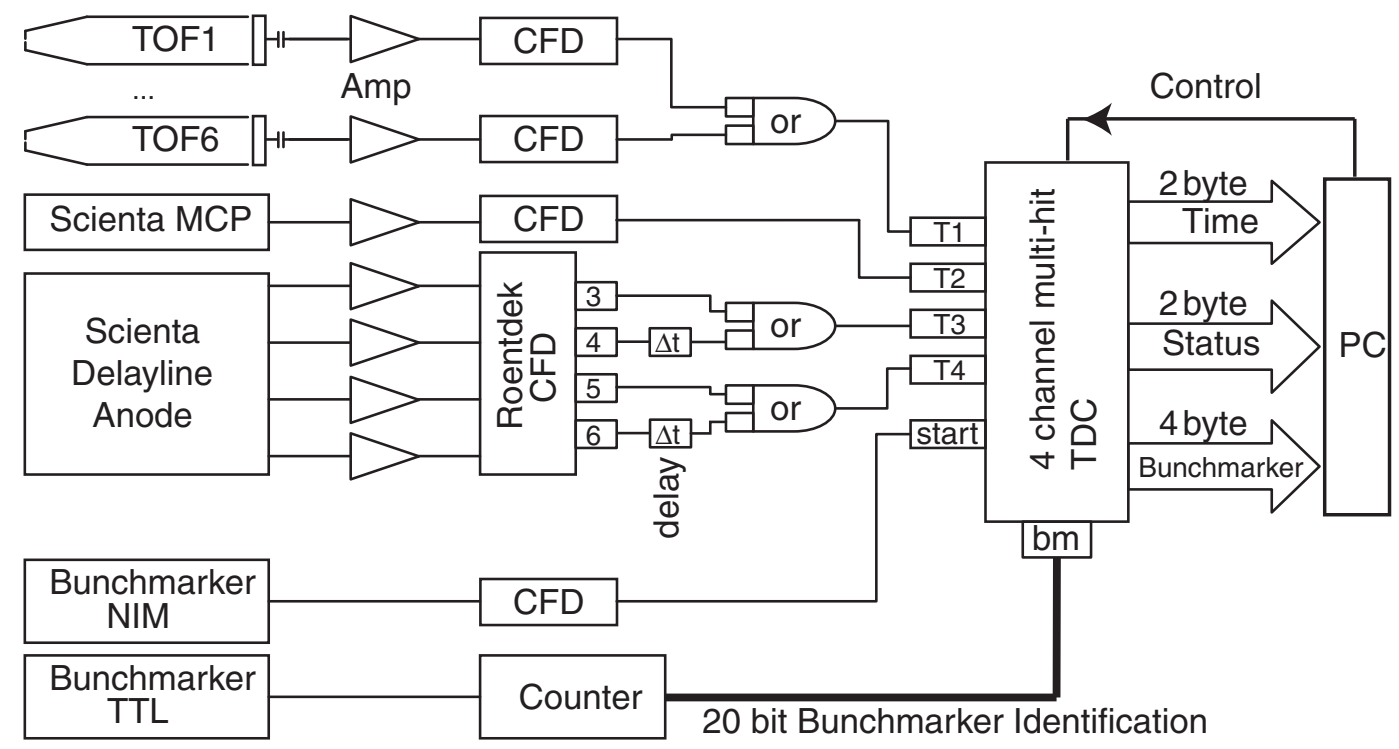

Fig. 5 

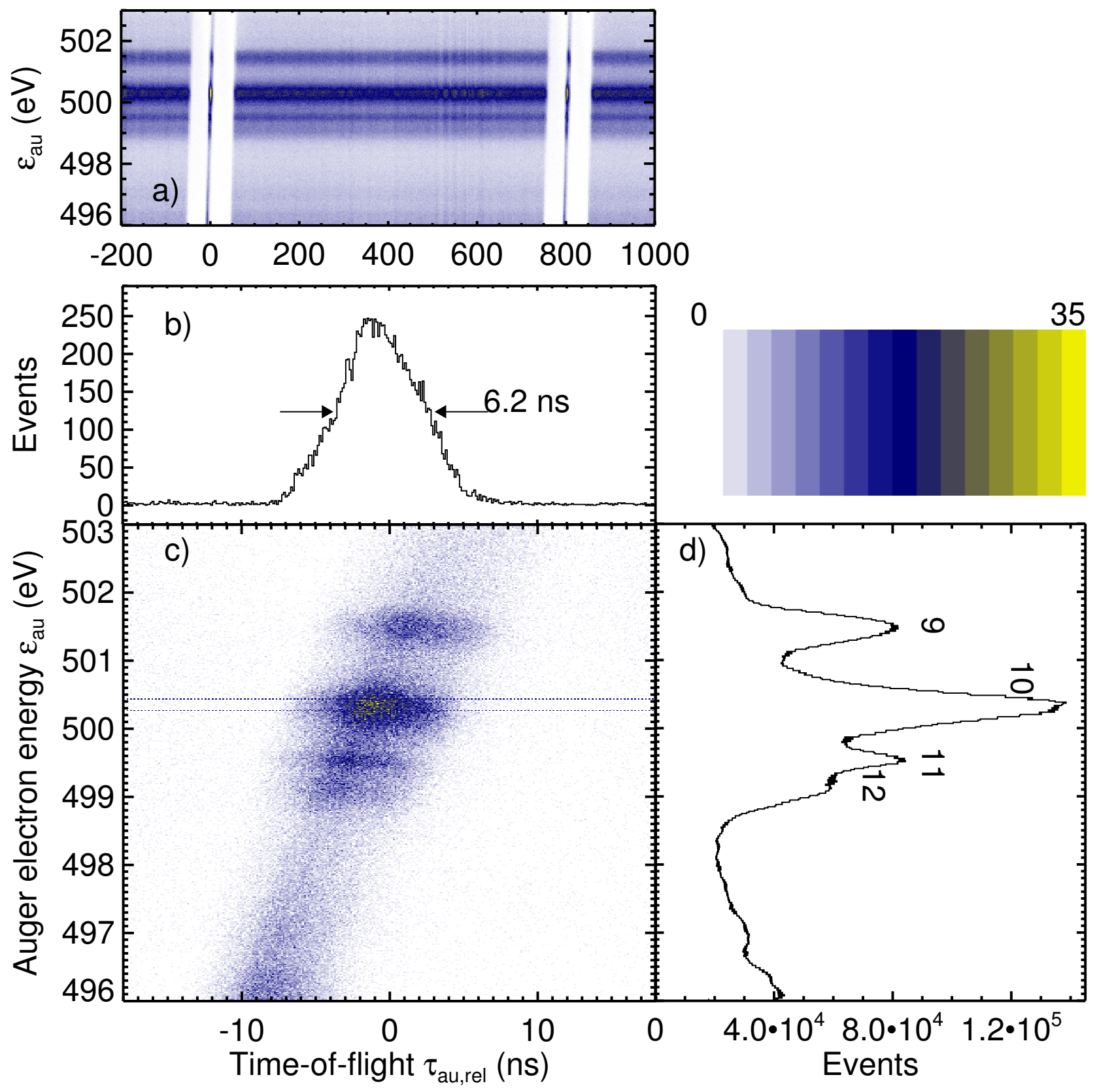

Fig. 


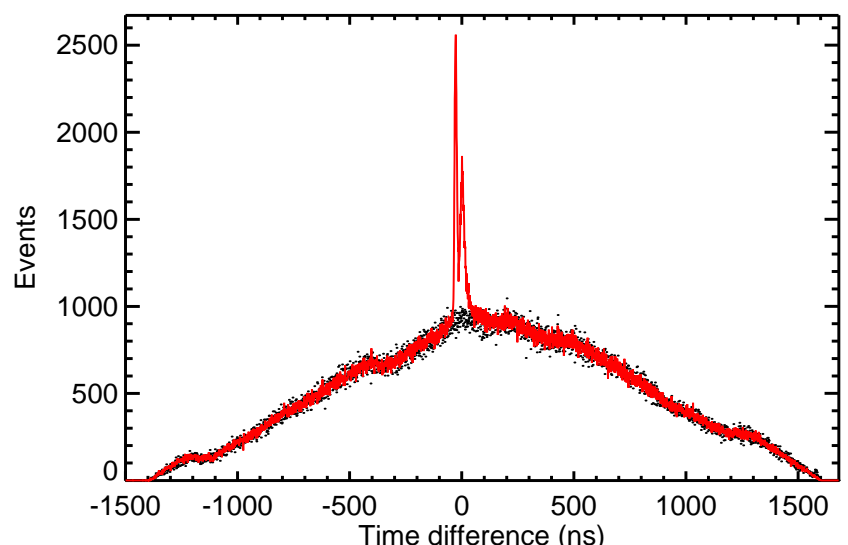

Fig. 7 


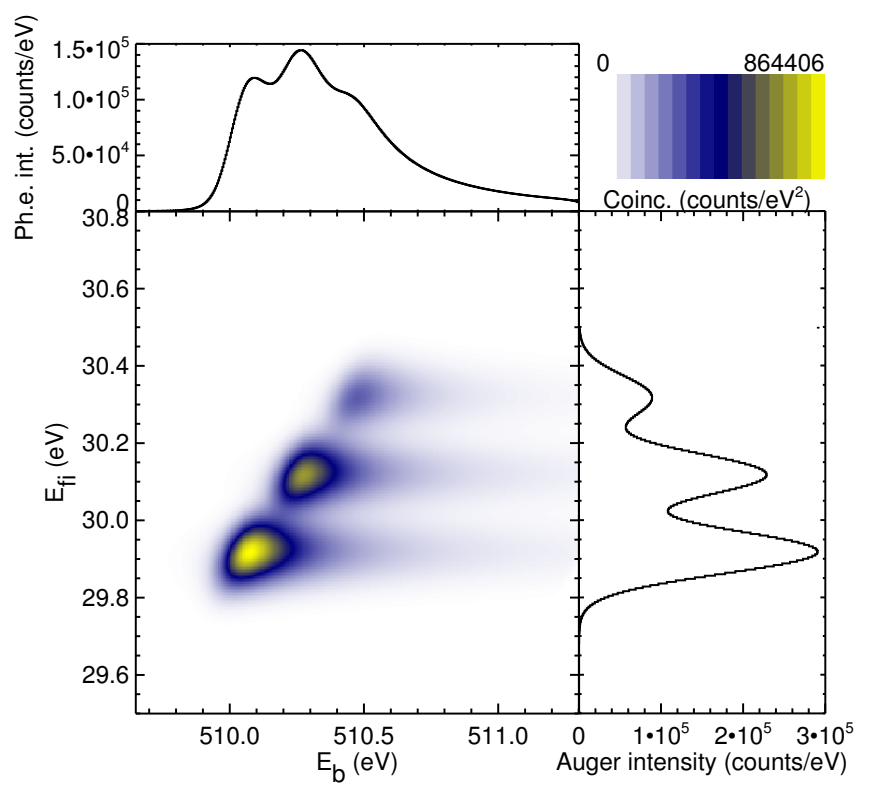

Fig. 8 


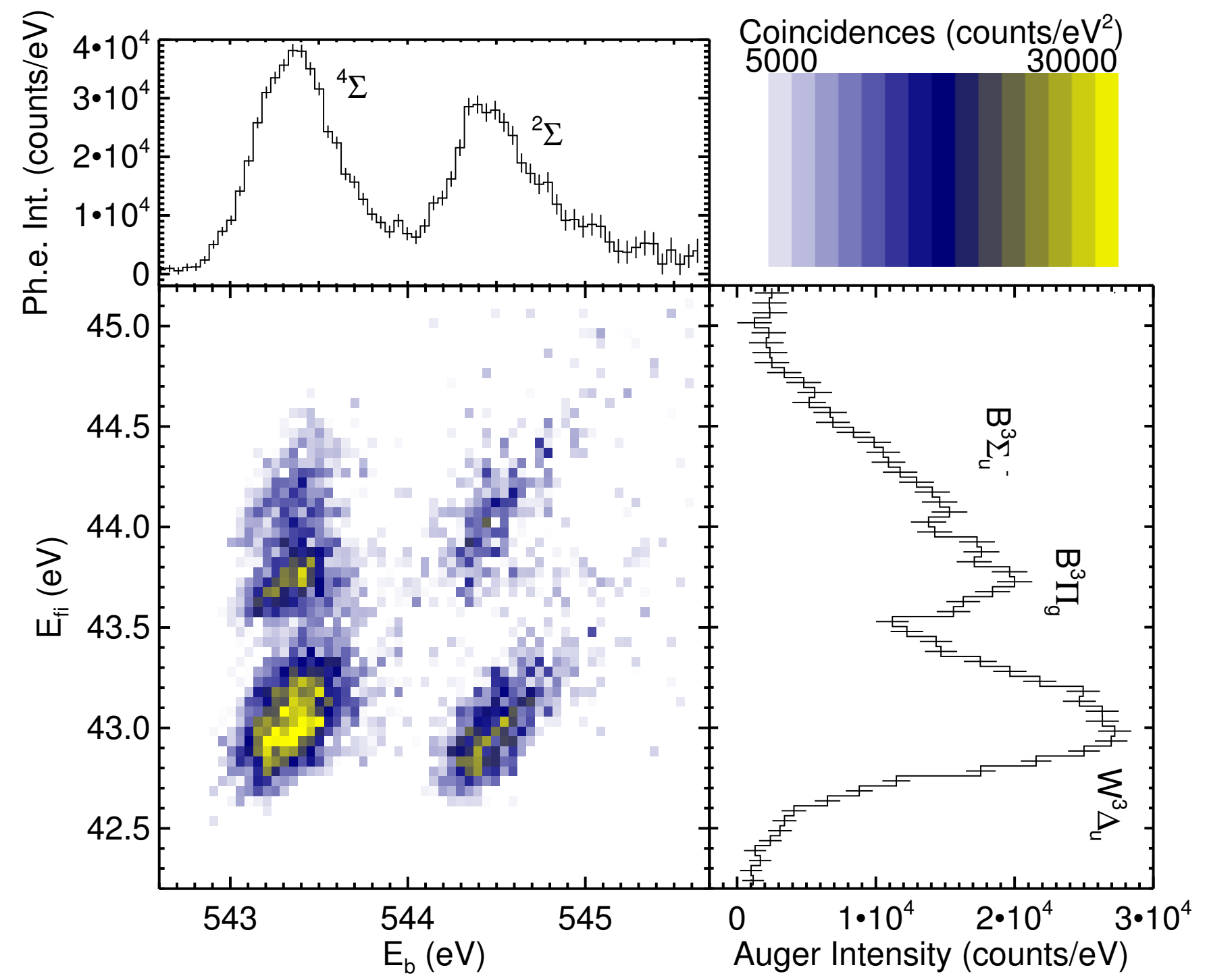

Fig. 9 


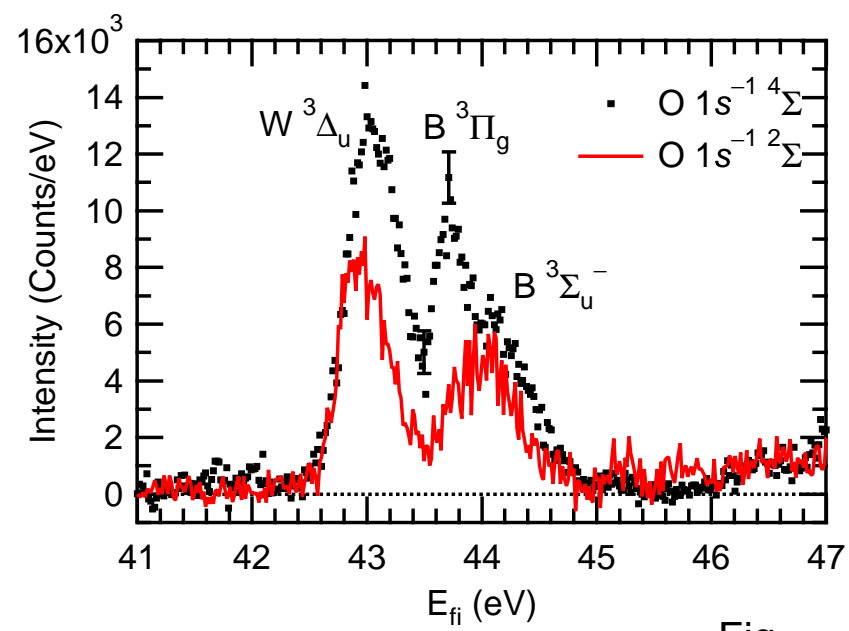

Fig. $\quad 10$ 